\title{
A Fast Logic Simulator Using a Look Up Table Cascade Emulator
}

Hiroki Nakahara

\author{
Tsutomu Sasao
}

\author{
Munehiro Matsuura
}

\author{
Depertment of Computer Science and Electronics \\ Kyushu Institute of Technology, Iizuka 820-8502, Japan
}

\begin{abstract}
This paper shows a new type of a cycle-based logic simulation method using a Look-Up Table (LUT) cascade emulator. The method first transforms a given circuit into LUT cascades through BDD (Binary Decision Diagram). Then, it stores LUT data to the memory of an LUT cascade emulator. Next, it generates the $\mathrm{C}$ code representing the control circuit of the LUT cascade emulator. And, finally, it converts the $C$ code into the execution code. This method is compared with a Levelized Compiled Code (LCC) simulator with respect to the simulation time and setup time. Although we used standard PC to simulate the circuit, experimental results show that this method is 12-64 times faster than the LCC.
\end{abstract}

\section{INTRODUCTION}

With the increase of the integration of LSIs, the time for the verification of the design increases. Thus, high-speed logic simulators are needed.

Logic simulators can be roughly divided into two types: event-driven simulators and cycle-based simulators. In an event-driven simulator, only the logic gates whose input signal change are evaluated. On the other hand, in a cycle-based logic simulator, the operation order of gates are determined statically beforehand, and all the logic values of the gates are evaluated for each clock cycle. Although the cycle-based logic simulator does not perform the timing verification, it is often faster than the event-driven simulator.

An LCC [1] is a kind of a cycle-based logic simulator using a general-purpose CPU. An LCC generates a program code for each gate of a logic circuit, and evaluates the circuit in a topological order from the inputs towards the outputs. An eventdriven simulator that emulates only logic gates whose outputs change has been developed [16]. It is at most two times faster than the LCC. In this paper, we will present a cycle-based logic simulator using an LUT cascade emulator. An LUT cascade emulator [2] consists of a control part, memories, and registers. Each register is connected to a programmable interconnection circuit, and the LUT cascade emulator evaluates the logic circuit stored in the memory. Murgai-Hirose-Fujita [11] also developed a logic simulator using large memories. Their method first converts a given circuit into a random logic network of single-output LUTs, then stores them in the memory, and finally evaluates the circuit by an event-driven logic simulator implemented by a hardware accelerator. In our method, we first convert the given circuit into a cascade rather than ran-
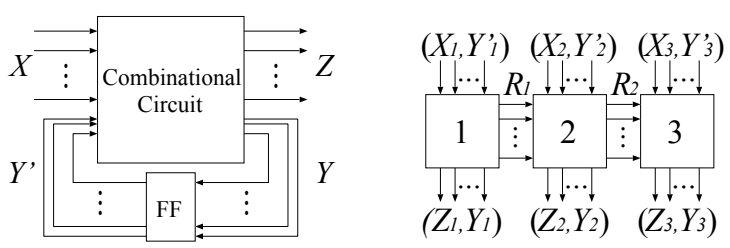

Fig. 1. A model for a sequential circuit. Fig. 2. LUT cascade.

dom logic, so the control part is simpler than Murgai-HiroseFujita's method. Also, our method uses multiple-output LUTs rather than single-output LUTs. In this paper, we consider the software-based logic simulation system where the LUT cascade emulator is simulated on a PC. Compared with the hardware-based logic simulator, logic simulator using a standard PC is much cheaper, and can be enhanced with the improvement of the performance of PCs.

\section{LUT CASCADE EMULATOR}

\section{A. LUT Cascade}

Fig. 1 shows a model for a sequential circuit, where $X$ denote inputs, $Z$ denote outputs, $Y$ denotes the inputs to flipflops, $Y^{\prime}$ denotes the outputs of flip-flops, and $|Y|$ denotes the number of state variables. We first consider an LUT cascade [3] that realizes the combinational part of the sequential circuit, then consider the LUT cascade emulator that emulates the LUT cascade.

An LUT cascade is shown in Fig. 2, where multiple-output LUTs (cells) are connected in series to realize a multipleoutput function. The wires connecting adjacent cells are called rails. Also, each cells may have external outputs in addition to the rail outputs. In this paper, $X_{i}$ denotes the external inputs to the $i$-th cell; $Y_{i}^{\prime}$ denotes the state inputs to the $i$-th cell; $Z_{i}$ denotes the external outputs of the $i$-th cell; $Y_{i}$ denotes the state outputs of the $i$-th cell; $R_{i-1}$ denotes the rail inputs to the $i$-th cell; and $R_{i}$ denotes the rail outputs from the $i$-th cell. We can obtain the LUT cascades by applying functional decomposition repeatedly to the BDD that represents the multiple-output function [4].

Definition 2.1 Let $\vec{X}=\left(x_{1}, x_{2}, \ldots, x_{n}\right)$ be the input variables, $\vec{Y}=\left(y_{1}, y_{2}, \ldots, y_{m}\right)$ be the output variables, and $\vec{f}=\left(f_{1}(\vec{X}), f_{2}(\vec{X}), \ldots, f_{m}(\vec{X})\right)$ be the corresponding out- 


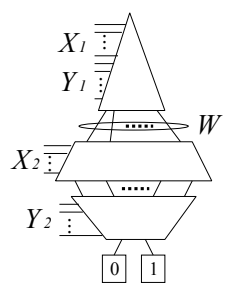

Fig. 3. BDD_for_CF.

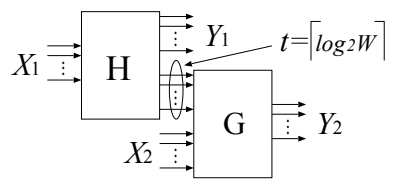

Fig. 4. Functional decomposition.

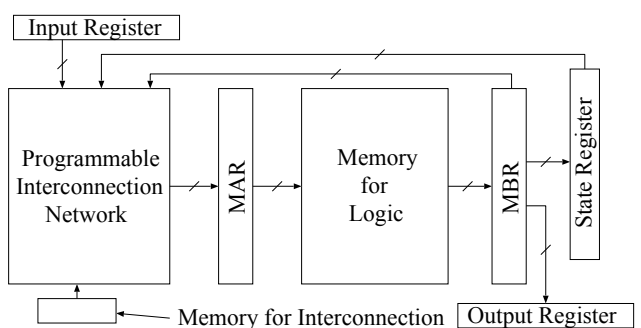

Fig. 5. LUT cascade emulator.

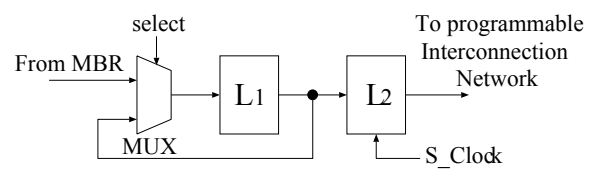

The characteristic function of an $n$-input $m$-output function is a two-valued logic function with $(n+m)$ inputs. It has input variables $x_{i}(i=1,2, \ldots, n)$, and output variables $y_{j}$ for output $f_{j}$. Let $B=\{0,1\}, \vec{a} \in B^{n}, \vec{F}=$ $\left(f_{1}(\vec{a}), f_{2}(\vec{a}), \ldots, f_{m}(\vec{a})\right) \in B^{m}$, and $\vec{b} \in B^{m}$. Then, the characteristic function satisfies the relation

$$
\vec{\chi}(\vec{a}, \vec{b})= \begin{cases}1 & \text { (when } \vec{b}=\vec{F}(\vec{a})) \\ 0 & \text { (otherwise) }\end{cases}
$$

Definition 2.2 A support variable of a function $f$ is a variable on which $f$ actually depends.

Definition 2.3 [5] The BDD_for_CF of a multiple-output function $\vec{f}=\left(f_{1}, f_{2}, \ldots, f_{m}\right)$ is the ROBDD [10] for the characteristic function $\vec{\chi}$. In this case, we assume that the root node is in the top of the BDD, and the variable $y_{i}$ is below the support variable of $f_{i}$, where $y_{i}$ is the variable representing $f_{i}$.

Definition 2.4 The width of the BDD_for_CF at height $k$ is the number of edges crossing the section of the graph between $x_{k}$ and $x_{k+1}$, where the edges incident to the same nodes are counted as one. Also, in counting the width of the BDD_for_CF, we ignore the edges that incident to the constant 0 node.

Let $X_{1}$ and $X_{2}$ be sets of input variables, $Y_{1}$ and $Y_{2}$ be sets of output variables, $\left(X_{1}, Y_{1}, X_{2}, Y_{2}\right)$ be the variable ordering of a BDD_for_CF for the multiple-output function $\vec{f}=$ $\left(f_{1}, f_{2}, \ldots, f_{m}\right)$, and $W$ be the width of the BDD_for_CF at the height $\left(X_{1}, Y_{1}\right)$ in Fig. 3. By applying functional decomposition to $\vec{f}$, we obtain the network in Fig. 4 , where the number of lines connecting two blocks is $t=\left\lceil\log _{2} W\right\rceil$ [4].

Theorem 2.1 [5] Let $\mu_{\max }$ be the maximum width of the $B D D_{-}$for_CF that represents an n-input logic function $\vec{f}$. If $u=\left\lceil\log _{2} \mu_{\max }\right\rceil \leq k-1$, then $\vec{f}$ can be realized by a circuit shown in Fig. 4, where $\left|X_{1}\right|=k$. By applying functional decompositions $s-1$ times, we have the cascade having the structure of Fig. 2.

\section{B. LUT Cascade Emulator}

Fig. 5 shows an LUT cascade emulator for a sequential circuit.
Fig. 6. Double rank flip-flop.

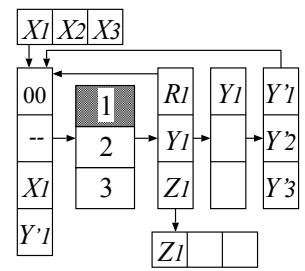

(a) Time $=1$

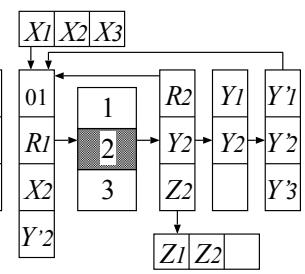

(b) Time $=2$

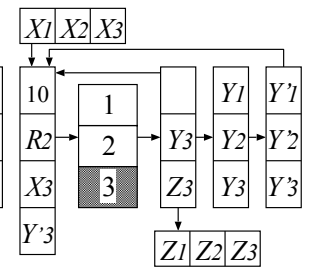

(b) Time $=3$
Fig. 7. Emulation of a sequential circuit

An LUT cascade emulator stores the cell data of an LUT cascade in the memory for logic. The address of cell data is calculated from inputs, state variables, and rail outputs of the preceding cell. The LUT cascade emulator reads the cell outputs from the memory for logic, and send them to the State Register and the Output Register. The Input Register stores the values of the primary inputs; the MAR (Memory Address Register) stores the address of the memory; the MBR (Memory Buffer Register) stores the outputs of the memory; the Programmable Interconnection Network connects the input register, the state register, and the MAR, also it connects the MBR and the MAR; the Memory for Interconnection stores data for the interconnections; and the Control Network generates necessary signals to obtain functional values.

To emulate a sequential circuit, the LUT cascade emulator stores state variables and output variables in the registers. Fig. 6 shows the Double-Rank Flip-Flop for the state register and the output register, where $L_{1}$ and $L_{2}$ are D-latches. Set the select signals to high when all the cells in a cascade are evaluated, and send the values into $L_{1}$ latches. When all the cascades are evaluated, the values of the state variables are sent to $L_{2}$ latches. This can be done by adding a pulse to S_Clock.

Example 2.1 Fig. 7 illustrates the emulation of the sequential circuit whose combinational part realizes the LUT cascade in Fig. 2.

At Time $=1$, to evaluate Cell 1, the two most significant bits of the address are set to $(0,0)$ to specify Page 1 . Also, the 
inputs to Cell $1 X_{1}$ and $Y_{1}^{\prime}$ are set to the lower address bits through the programmable interconnection network. By reading Page 1, the outputs of Cell 1 are sent to MBR. For the outputs that become the primary output $Z_{1}$, store it in the output register, while for the output that becomes the state output $Y_{1}$, store it in the state register (Fig. 7(a)).

At Time $=2$, to evaluate Cell 2, the two most significant bits of the address are set to $(0,1)$ to specify Page 2 . Also, the outputs of Cell $1, R_{1}$ are connected to the middle address bits, and the input variables of Cell $2 X_{2}$ and $Y_{2}^{\prime}$ are set to the lower address bits through the programmable interconnection network. By reading Page 2, the outputs of Cell 2 are sent to MBR. For the output that becomes the primary output $Z_{2}$, store it in the output register, while for the output that becomes the state output $Y_{2}$, store it in the state register (Fig. 7(b)).

At Time $=3$, to evaluate Cell 3, the two most significant bits of the address are set to $(1,0)$ to specify Page 3 . Also, the outputs of Cell 2, $R_{2}$ are connected to the middle address bits, and the input variables of Cell $3 X_{3}$ and $Y_{3}^{\prime}$ are set to the lower address bits through the programmable interconnection network. By reading Page 3, the outputs of Cell 3 are sent to MBR. For the output that becomes the primary output $Z_{3}$, store it in the output register, while for the output that becomes state output $Y_{3}$, store it in the state register (Fig. 7(c)).

When all the cells of cascades are evaluated, Control Network sends a pulse to S_Clock of the state register and the output register, and the values of state outputs $Y_{1}, Y_{2}, Y_{3}$ are sent to the $L_{2}$ latches. Also, the values of the output register $Z_{1}, Z_{2}, Z_{3}$ are sent to the primary outputs.

(End of Example)

\section{SyNTHESIS OF THE LUT CASCADE EMULATOR}

\section{A. Partition of the outputs}

When the number of outputs is large, we partition the outputs into groups, and realize a cascade for each group independently. Usually, the BDD_for_CF for all the outputs are too large to construct. Even if the BDD_for_CF is constructed, it can be too large to be realized by an LUT cascade. Also, constructing a single BDD_for_CF for all the outputs is inefficient, since the optimization of a large BDD_for_CF is time consuming.

In order to construct as small BDD_for_CFs as possible, we partition the outputs so that each group has a small number of support variables.

Definition 3.5 Let $F=\left\{f_{1}, f_{2}, \ldots, f_{m}\right\}$ be the set of the outputs functions, $G \subseteq F$, and $f_{i} \in F-G$. Then, the similarity of the output $f_{i}$ with $G$ is defined as follows:

$$
\operatorname{Similarity}(i, G, F)=\left|\operatorname{Sup}\left(f_{i}\right) \cap \operatorname{Sup}(G)\right|,
$$

where $\operatorname{Sup}(F)$ denotes the set of support variables of $F$.

\footnotetext{
Algorithm 3.1 (Partition the Outputs and Construction of $\left.B D D_{-} f_{0} \_C F\right)$

Let $F=\left\{f_{1}, f_{2}, \ldots, f_{m}\right\}$ be the set of $m$ logic functions,
}

$\mathcal{Z}=\left\{G_{1}, G_{2}, \ldots, G_{r}\right\}$ be the set of subset of output functions after partitioning, $r$ be the number of partitions, and Th_node be the threshold of the number of nodes.

1. $\mathcal{Z} \leftarrow \phi, r \leftarrow 0$.

2. While $F \neq \phi$, do Steps (a)-(d).

(a) Node $\leftarrow 0, G_{r} \leftarrow \phi$.

(b) While Node $\leq$ Th_node, $F \neq \phi$, and $G_{r}$ is cascade realizable, do Steps i-iii.

i. Select $f_{i}$ with the maximum $\operatorname{Similarity}\left(i, G_{r}, F\right)$. If $G_{r}=\phi$, then select $f_{i}$ that has the largest support.

ii. $G_{r} \leftarrow G_{r} \cup\left\{f_{i}\right\}, F \leftarrow F-\left\{f_{i}\right\}$.

iii. Construct BDD_for_CF that realizes $G_{r}$, and Node $\leftarrow$ (the number of nodes).

(c) If $G_{r}$ is not cascade realizable, then $G_{r} \leftarrow G_{r}-$ $\left\{f_{i}\right\}, F \leftarrow F \cup\left\{f_{i}\right\}$.

(d) $\mathcal{Z} \leftarrow \mathcal{Z} \cup\left\{G_{r}\right\}, r \leftarrow r+1$.

\section{Terminate.}

This method merges outputs into a group while a cascade is realizable and the number of nodes in the BDD is equal to or less than the threshold. Algorithm 3.1 partitions the outputs into the groups so that the resultant BDDs are small enough.

\section{B. Memory Packing}

By Alogrithm 3.1, a given multiple-output function is represented by a set of BDD_for_CFs. Then, the LUT cascades are constructed, and LUT data is allocated into the memory of the LUT cascade emulator.

Example 3.2 Fig. 8 shows the LUT cascade consisting of 4input cells. Fig. 9(a) illustrate the memory map of cell data, where the memory has 6-bit address inputs, and each word consists of four bits. The dark parts in the figure are unused, and $P_{i}$ denotes the page number.

(End of Example)

In Example 3.2, each cell data are stored in a separate page of the memory. The data of a cell must be stored in the same page, and must be read simultaneously. However, if there are any extra space in the same page, multiple cell data can be stored in the same page. This method to reduce the memory area is called memory-packing [6].

Example 3.3 In Fig. 9(a), by storing the cell data $r_{5}$ and $z_{1}$ to Page 1, we have the memory map in Fig. 9(b), where a half of the memory is enough to store all the data. (End of Example)

\section{LOGiC Simulation USING AN LUT CASCADE EMULATOR}

\section{A. Generation of the execution code for the simulation}

Fig. 10 shows the data flow of the logic simulation system using LUT cascade emulator. 


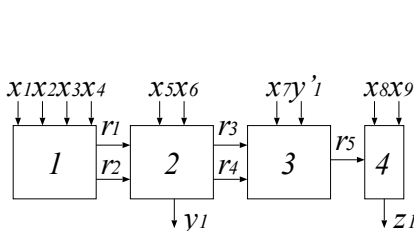

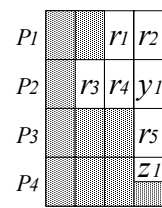

(a)

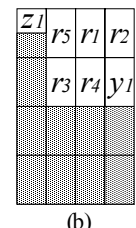

Fig. 9. Example of memory-packing.
Fig. 8. Example of LUT cascade.

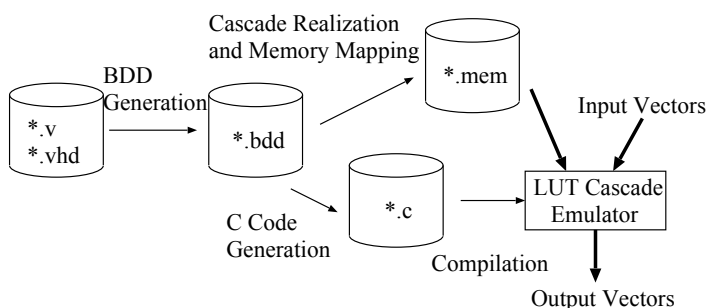

Fig. 10. Flow of data for the logic simulation system using LUT cascade emulator.

First, it converts the Verilog-VHDL code describing the given circuit into shared-BDDs [9]. Then, it reduces the number of nodes of BDDs by changing variable orders [7]. Next, it generates the LUT cascades from BDDs using the functional decomposition described in Chapter 2, and it maps them to the memory of the LUT cascade emulator. Also, it generates the $\mathrm{C}$ code that simulates the control circuit of the LUT cascade emulator. Next, it complies the $\mathrm{C}$ code into the execution code for simulation of the LUT cascade emulator. And, finally the simulator evaluates the output of the given circuit, by using the memory map of the LUT cascade emulator.

\section{B. Program code that simulates the LUT cascade emulator}

This system generates the program code that describes the following operations.

Step 1 Set the input register, and initialize the state register.

An input value is set to the input register. Also, the value of the state register is initialized.

Step 2 Evaluate of each cell.

Step 2.1 Simulate the programmable interconnection network.

The address of the memory for logic is generated from the values of the input register, the state input register, the MBR, and a page address.

Step 2.2 Read the memory for logic.

The content of the memory for logic is read using the address generated by Step 2.1.

Step 2.3 Distribute output values of the memory for logic. The values read by Step 2.2 are sent to the output register and the state register.

Step 3 Perform the state transitions.

The output values of the state register is updated by S_Clock.
Assigning each memory output to each register consumes CPU time. Fortunately, the memory outputs are stored in the order of primary outputs, state outputs, and rail outputs. For a 32-bit processor, we can evaluate up to 32 outputs at a time. To obtain required outputs, we shift the memory outputs covered by a mask, and assign to a 32-bit variable. In this way, we can evaluate the multiple outputs simultaneously. Also, there is an additional merit for performing the state transition. Let $|Y|$ be the number of state variables for given logic function, then the number of evaluations for the state transition is $\left\lceil\frac{|Y|}{32}\right]$ for a 32-bit machine.

Since cascades have much fewer signal lines than the original circuit, the compilation time for cascades are much shorter than that of the conventional LCC method.

\section{Analysis of Simulation Time}

When the LUT cascade emulator is implemented on a dedicated hardware [2], the evaluation time is proportional to the number of cells. However, when the LUT cascade emulator is implemented on a standard PC, we need extra time since the inputs and outputs of a cell are evaluated sequentially.

To do high-speed simulation for the LUT cascade emulator on a PC, we have to consider two different objects:

a. Reduction of the number of cells.

This can be done by increasing the number of inputs of each cell. However, the increase of the number of inputs of each cell also increases the evaluation time per cell.

b. Reduction of the number of cell inputs.

This decreases the evaluation time per cells, but increases the number of cells.

To find the best strategy, we did following experiments. We implemented 10 MCNC benchmark functions [8] on the LUT cascade emulator. By changing the maximum number of inputs for cells, we obtained the average number of cell inputs, the number of cells, and the execution time of the LUT cascade emulator. Fig. 11 shows the experimental results, where the horizontal axis denotes the maximum number of cell inputs; 0 denotes the lower bound on the maximum number of inputs of cells, that is $\left\lceil\log _{2} \mu_{\max }\right\rceil+1$; the vertical axis denotes the ratios of the number of cells, the number of the average cell inputs, and simulation time. We set 1.00 to the ratios when the number of cell inputs is $\left\lceil\log _{2} \mu_{\max }\right\rceil+1$.

Fig. 11 shows that the simulation time increases with the number of cell inputs. To compute the address of the memory for logic, we need CPU time. This CPU time increase with the number of inputs of a cell. Therefore, our strategy is to reduce the number of cell inputs in the LUT cascade emulator.

\section{EXPERIMENTAL RESULTS}

\section{A. Comparison with LCC}

We simulated selected MCNC benchmark functions by LUT cascade emulator and LCC on a same PC. Table I shows the 


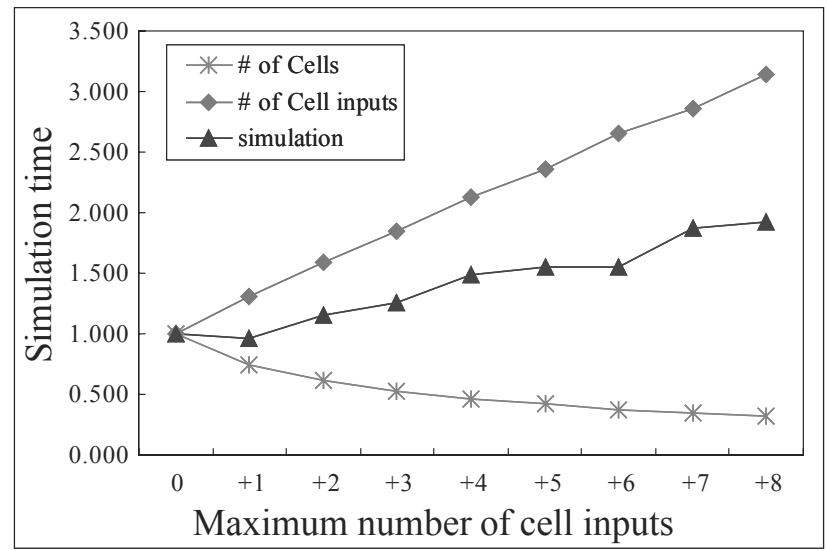

Fig. 11. Relation between the maximum number of cell inputs and simulation time.

experimental results. Name denotes the name of benchmark function; In denotes the number of inputs; Out denotes the number of outputs; State denotes the number of state variables; Cas denotes the number of LUT cascades; Cell denotes the total number of cells; and Mem denotes the amount of memory (Mega Bits). Also, EXT.in denotes the average number of external inputs to cells; P.out denotes the total number of cells with external output(s); and S.out denotes the total number of cells with state output(s). Sim denotes the evaluation time (sec). In order to obtain the raw evaluation time for the simulation, we generated the one million random test vectors, and evaluated the time excluding the time for reading and writing vectors. Setup denotes the setup time $(\mathrm{sec})$ for a simulation. Setup of LCC is the time for the C code generation and the compilation, while Setup of the LUT cascade emulator is the time for BDD generation, LUT cascades synthesis, memory mapping, $\mathrm{C}$ code generation, and the compilation. Literals denotes the total number of literals in expressions of lines of the $\mathrm{C}$ code generated by the LCC. Ratio denotes that of the simulation setup time or that of the simulation execution time (LCC/LUT cascade emulator). To produce the executable code for LCC, we used gcc compiler with optimization option -O3. Also, we generated program codes for LUT cascade emulator, and compiled them with the same conditions as LCC. In the experiments, we used an IBM PC/AT compatible machine, Pentium4 Xeon 2.8GHz, L1 Cache: 8KB, L2 Cache: 512KB, Memory: 4GByte, and OS: Redhad (Linux 7.3). For the benchmark function $c l m a$, it has many redundant primary inputs, primary outputs, and logic gates. For pre-processing, we simplified the Verilog-VHDL descriptions of benchmark functions by using Quartus II version 5.0 [13]. Note that, the time for the pre-processing is not included in the setup time.

Table I shows that the LUT cascade emulator is 12 - 64 times faster than the LCC. Also, the setup speed for the LUT cascade emulator is 1.20 - 5.22 times faster than the LCC, except for s5378 and clma.

The number of operations in the LUT cascade emulator is

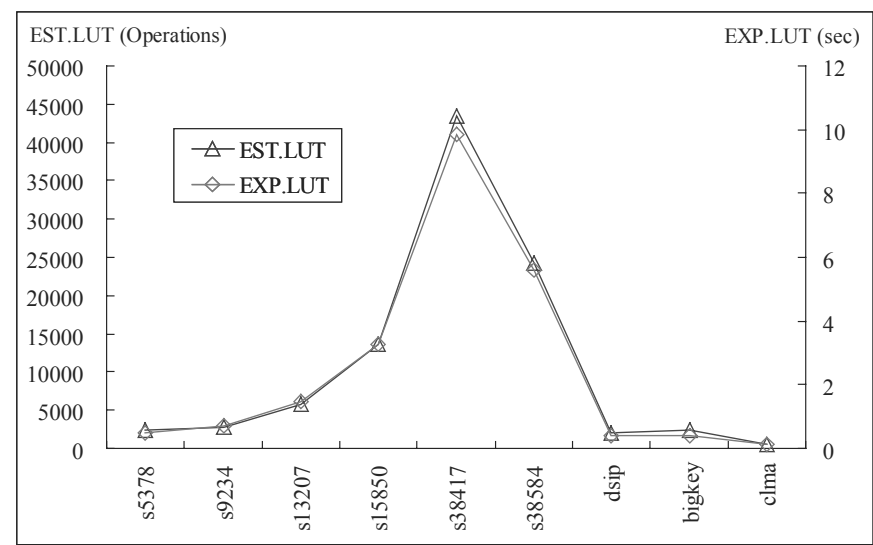

Fig. 12. Simulation time for LUT cascade emulator.

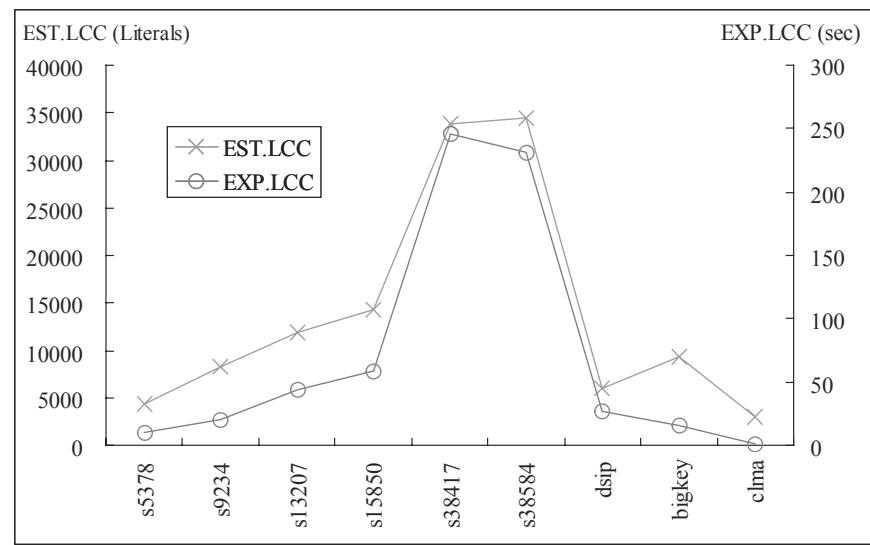

Fig. 13. Simulation time for LCC.

estimated as follows:

$$
\begin{aligned}
\text { EST.LUT }= & \text { EXT.in } \times \text { Cell } \\
& + \text { Cell }+ \text { P.out }+ \text { S.out }+ \text { Rail },
\end{aligned}
$$

where Rail $=$ Cell - Cas. The first term of expression (2) corresponds to the setup time of all the external inputs of the cells; the second term corresponds to the access time to the memory for logic; the third term corresponds to the setup time for the output register; the fourth term corresponds to the setup time for the state register; and the last term corresponds to the setup time for the rail inputs.

In Fig. 12, the right vertical axis denotes the experimental value EXP.LUT (sec), and the left vertical axis denotes the estimated number of operations EST.LUT. Also, we conjecture that Literals is proportional to the simulation time for LCC. In Fig. 13, the right vertical axis denotes the experimental value EXP.LCC $(\mathrm{sec})$, and the left vertical axis denotes the estimated number of literals EST.LCC = Literals. Figs. 12 and 13 show that the EXP.LUT and EXP.LCC can be estimated from EST.LUT and EST.LCC, respectively.

In Figs. 12 and 13, note that EXP.LUT is smaller than EXP.LCC, while EST.LUT (number of operations) is larger than EST.LCC (number of literals) for some functions. This may look strange. To see the reason, we converted the $\mathrm{C}$ codes 
TABLE I

RESULTS OF REALIZATION OF BENCHMARK FUNCTIONS

\begin{tabular}{|c|c|c|c|c|c|c|c|c|c|c|c|c|c|c|c|c|}
\hline \multirow[t]{2}{*}{ Name } & \multirow[t]{2}{*}{ In } & \multirow[t]{2}{*}{$\overline{\text { Out }}$} & \multirow[t]{2}{*}{ State } & \multicolumn{8}{|c|}{ LUT cascade emulator } & \multicolumn{3}{|c|}{$\mathrm{LCC}$} & \multicolumn{2}{|c|}{ Ratio } \\
\hline & & & & Cas & Cell & $\begin{array}{c}\text { Mem } \\
\text { [Mbit] }\end{array}$ & EXT.in & P.out & S.out & $\begin{array}{l}\text { Setup } \\
{[\mathrm{sec}]}\end{array}$ & $\begin{array}{c}\text { Sim } \\
{[\mathrm{sec}]}\end{array}$ & Literals & $\begin{array}{l}\text { Setup } \\
\text { [sec] }\end{array}$ & $\begin{array}{l}\mathrm{Sim} \\
{[\mathrm{sec}]}\end{array}$ & Setup & Sim \\
\hline s5378 & 35 & 49 & 164 & 40 & 543 & 0.82 & 2.39 & 105 & 28 & 14.59 & 0.49 & 4424 & 10.26 & 10.46 & 0.70 & 21.35 \\
\hline s9234 & 36 & 39 & 211 & 44 & 599 & 0.91 & 2.63 & 26 & 120 & 14.68 & 0.71 & 8220 & 41.01 & 20.64 & 2.79 & 29.07 \\
\hline s13207 & 62 & 152 & 638 & 93 & 1245 & 3.28 & 2.27 & 109 & 390 & 31.09 & 1.46 & 11954 & 83.04 & 43.76 & 2.67 & 29.97 \\
\hline s 15850 & 77 & 150 & 534 & 105 & 3370 & 8.95 & 1.95 & 115 & 338 & 79.25 & 3.25 & 14328 & 115.72 & 58.71 & 1.46 & 18.06 \\
\hline s38417 & 28 & 106 & 1636 & 389 & 9411 & 45.36 & 2.55 & 60 & 964 & 763.07 & 9.87 & 33769 & 917.40 & 245.63 & 1.20 & 24.89 \\
\hline s38584 & 38 & 304 & 1426 & 270 & 5118 & 16.90 & 2.55 & 232 & 956 & 159.09 & 7.61 & 34485 & 830.27 & 230.76 & 5.22 & 30.33 \\
\hline dsip & 229 & 197 & 224 & 45 & 473 & 6.60 & 1.96 & 108 & 115 & 10.90 & 0.42 & 5959 & 26.39 & 27.08 & 2.42 & 64.48 \\
\hline bigkey & 263 & 197 & 224 & 48 & 541 & 3.76 & 1.97 & 171 & 121 & 11.74 & 0.42 & 9262 & 27.58 & 16.26 & 2.35 & 38.71 \\
\hline clma & 101 & 81 & 33 & 9 & 45 & 0.16 & 3.28 & 27 & 21 & 3.59 & 0.11 & 2994 & 2.87 & 1.37 & 0.79 & 12.45 \\
\hline
\end{tabular}

of the LUT cascade emulator and the LCC into the assemblycodes, and analyzed them. The size of the assembly-code for the LCC is several times larger than EST.LCC. This is because the LCC compiler generates extra codes to evaluate the negative literals and the logic gates, and to produce the output signals. On the other hand, the size of the assembly-code for LUT cascade emulator does not depend on EST.LUT, and is much smaller than EST.LUT. In the LCC, it's operands frequently move between register and memory. For the gate with fanouts, the LCC stores the output values of the gate into a variable temporarily, and uses it as the input of two or more gates. On the other hand, the LUT cascade emulator uses only the rail values in the single register variable. Therefore, only the input and output register and the memory for logic requires memory references. Experimental results show that the simulator based on an LUT cascade emulator is 12-64 times faster than the LCC. One reason for this is the difference of the representations: the cascade has many fewer signals than the random logic network. Another reason is due to the CPU architecture of the PC. The access time of the data in the main memory is about 200 times longer than one in the L1 cache. So, the CPU time heavily depends on the frequency of cache miss. In the case of the LCC simulator, the circuit data and control are mixed, and the instruction data is too large to be stored in the data cache. On the other hand, in the case of an LUT cascade emulator, the cascade data and control are separated. Control data is in the instruction cache, while the cascade data is in the data cache. Thus, we can expect fewer cache miss in the LUT cascade emulator.

\section{B. Comparison with Commercial Tools}

We compare the our method with two commercial simulators: Super-FinSim [14] version 6.2.9 and ModelSim [15] Altera-Edition (AE) 6.0c. ModelSim (AE) is an event-driven logic simulator, bundled with Quartus II. It supports functional simulation involving the 'zero-delay'. To obtain the evaluation time for the functional simulation, first, we generate a verilog code for a testbench including $10^{4}$ test vectors. Then, we compiled the verilog codes, representing benchmark circuit and testbench, using a command 'vlog [benchmark name.v]' with option '+notimingcheck' and 'no_notifier'. Next, we evaluate the simulated time using commands which are 'vsim -c' and
TABLE II

RESULTS OF COMPARISON WITH COMMERCIAL TOOLS

\begin{tabular}{|l|r|r|r|r|r|}
\hline \multirow{2}{*}{ Name } & \multicolumn{3}{|c|}{ Simulation time } & \multicolumn{2}{|c|}{ Ratio } \\
\cline { 2 - 6 } & FinSim & ModelSim & $\begin{array}{c}\text { LUT Cascade } \\
\text { Emulator }\end{array}$ & v.s.FinSim & v.s.ModelSim \\
\hline s5378 & 1.45 & 35.77 & 0.09 & 16.11 & 397.44 \\
\hline s9234 & 1.73 & 44.72 & 0.11 & 15.73 & 406.55 \\
\hline s13207 & 6.59 & 46.50 & 0.17 & 38.76 & 273.53 \\
\hline s15850 & 14.84 & 68.63 & 0.58 & 25.59 & 118.33 \\
\hline s38417 & 17.93 & 72.70 & 3.28 & 5.47 & 22.16 \\
\hline s38584 & 58.21 & 155.84 & 1.76 & 33.07 & 88.55 \\
\hline dsip & 14.31 & 30.42 & 0.16 & 89.44 & 190.13 \\
\hline bigkey & 2.99 & 37.28 & 0.08 & 37.38 & 466.00 \\
\hline clma & 41.13 & 88.86 & 0.09 & 457.00 & 987.33 \\
\hline
\end{tabular}

'run 10000'. Super-FinSim supports both the enhanced cycle simulation (ECS) and the event-driven simulation at the same time. To compare the our method with the ECS, we generated the testbench similar to ModelSim, and we complied verilog codes using a command 'finvc' with option '+delay_mode_zero', '-dsm com', '-ol 1', '-acc', '-fastgate', '+notimingcheck', and '+no_notifier'. Then, we built an execution file using the command 'finbuild', and evaluated the simulation time. We used Microsoft Visual C++ version 6.0 for SuperFinSim. In the experiments, we used an IBM PC/AT compatible machine, Pentium4 3.06GHz, L1 Cache: 8KB, L2 Cache 512KB, Memory 2GByte, and OS: Windows XP Professional SP2. To realize the LUT cascade emulator for Linux on Windows, we used gcc compiler version 3.2 on the cygwin.dll version 1.3.22 which emulates Linux API using Windows API.

Table II compares the results. Simulation time denotes the actual simulation time including the time for reading and writing $10^{4}$ vectors; and Ratio denotes that of the simulation execution time (Super-FinSim / LUT cascade emulator) and (ModelSim / LUT cascade emulator), respectively.

Table II shows that the LUT cascade emulator is 22.16987.33 times faster than ModelSim, and 5.47-457.00 times faster than FinSim.

Especially, for benchmark clma, our method is hundreds times faster than commercial tools. clma is a special benchmark circuit with redundant input-and-output signals and redundant gates. In order to perform a high-speed simulation, our method converted the circuit into BDDs to remove these 


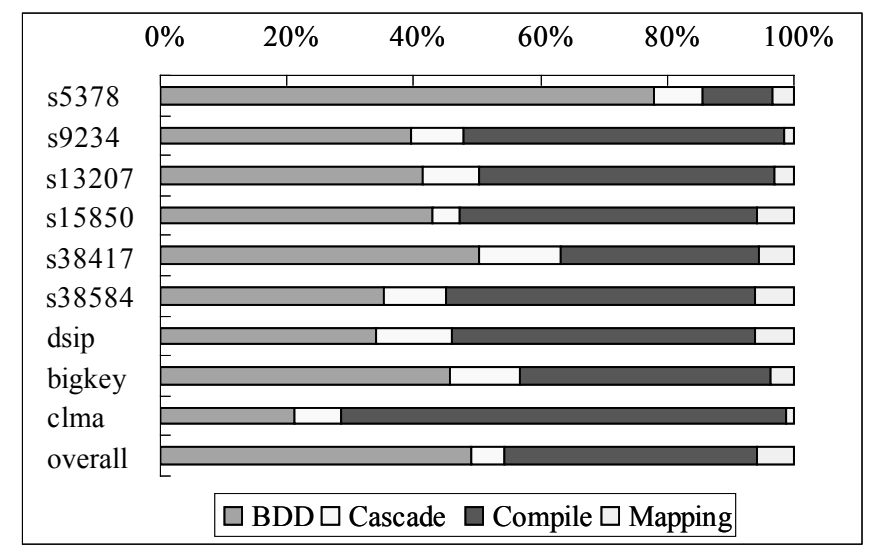

Fig. 14. Percentage of each process for setup time.

redundancy. However, ModelSim and FinSim used original circuits, so the redundancy of circuits affected the simulation time.

\section{Setup Time for the LUT Cascade Emulator}

Fig. 14 shows the percentage of each process of the setup time for the LUT cascade emulator. The labels of the vertical axis show function names. The horizontal axis shows the percentage of the processing time for BDD for $\mathrm{CF}$ generation $(B D D)$; LUT cascade synthesis (cascade); $\mathrm{C}$ code generation and compilation (compile); and memory mapping (mapping). overall denotes the average percentage of each process. Fig. 14 shows that BDD generation and compilation consume most of the CPU time. For BDD generation, optimization of variable orders for BDDs consumes most of the CPU time. Reduction of the code size, e.g., reduction of the number of cells, is effective to reduce the compilation time. Increase of cells increases the simulation setup time. The time for memory mapping increases with the number of cell inputs. The amount of memory for each cell is $2^{k} \cdot u$, where $k$ is the number of inputs of a cell, and $u$ is the number of outputs of a cell. Therefore, the number of cell inputs influences the total amount of data. In our experiment, since the average number of cell inputs is small, the memory mapping time did not influence the setup time.

\section{CONCLUSION AND COMmEnTS}

In this paper, we showed a cycle-based logic simulator using the LUT cascade emulator running on a standard PC. This method converts the circuit into LUT cascades. Then, it stores the LUT data in the memory of the LUT cascade emulator. Next, it generates the program code for the control circuit of the LUT cascade emulator. The program code is suitable for logic simulation on a standard PC due to the better memory reference patterns. Experimental results using benchmark functions show that this method is 12-64 times faster than LCC on a standard PC.

Our method converts the circuit into BDDs, where the sizes of BDDs representing the circuits are limited due to the avail- able memory. To avoid this limitation, we partition the output functions into groups. However, when the BDD representing a single output is excessively large, our method fails to perform the emulation.

One of the future projects is to derive a partition method for the circuit and to represent the circuits by smaller BDDs.

\section{ACKNOWLEDGMENTS}

This research is partly supported by Japan Society for the Promotion of Science (JSPS), MEXT, and Kitakyushu Innovative Cluster.

\section{REFERENCES}

[1] M. Abramovici, M. A. Breuer, and A. D. Friedman, Digital Systems Testing and Testable Design, Wiley-IEEE Press; Rev. Print edition, Sept. 1994.

[2] T. Sasao, Y. Iguchi, and M. Matsuura, "LUT cascades and emulators for realizations of logic functions," RM2005, Tokyo, Japan, Sept. 5-6, 2005, pp.63-70.

[3] T. Sasao, M. Matsuura, and Y. Iguchi, "A cascade realization of multiple-output function for reconfigurable hardware," IWLS-2001, Lake Tahoe, CA, June 12-15,2001, pp.225-300.

[4] T. Sasao, and M. Matsuura, "A method to decompose multiple-output logic functions," Proc. Design Automation Conference, San Diego, CA, USA, June 2-6, 2004, pp.428-433.

[5] P. Ashar, and S. Malik, "Fast functional simulation using branching programs,” ICCAD’95, Nov.1995, pp.408-412.

[6] T. Sasao, M. Kusano, and M. Matsuura, "Optimization methods in look-up table rings," IWLS-2004, June 2-4, 2004, Temecula, California, USA, pp.431-437.

[7] R. Rudell, "Dynamic variable ordering for ordered binary decision diagrams," ICCAD'93, pp. 42-47, 1993.

[8] S. Yang, Logic synthesis and optimization benchmark user guide version 3.0, MCNC, Jan. 1991.

[9] S. Minato, N. Ishiura, and S. Yajima, "Shared binary decision diagram with attributed edges for efficient boolean function manipulation," Proc. Design Automation Conference, June 1991, pp.52-57.

[10] R. E. Bryant, "Graph-based algorithms for boolean function manipulation,” IEEE Transact. Comput., C-35, Aug.1986, pp.677-691.

[11] R. Murgai, F. Hirose, and M. Fujita, "Logic synthesis for a single large look-up table,” ICCD1995, pp.415-424, Oct. 1995.

[12] P. C. McGeer, K. L. McMillan, A. Saldanha, A. L. SangiovanniVincentelli, and P. Scaglia, "Fast discrete function evaluation using decision diagrams," ICCAD'95, pp.402-407, Nov. 1995.

[13] Altera: http://www.altera.com/

[14] Fintronic USA, Inc.: http://www.fintronic.com/

[15] Mentor Graphics Corporation: http://www.model.com/

[16] P. M. Maurer, "The Inversion algorithm for digital simulation," ICCAD'94, pp.258-261, Nov. 1994. 